\title{
Separatrix formalism for superconducting linear accelerators
}

\author{
Yu. Senichev, A. Bogdanov, and R. Maier \\ FZJ, Institute of Nuclear Physics, D-52425, Juelich, Germany
}

(Received 7 October 2003; published 3 December 2003)

\begin{abstract}
Superconducting cavities provide high accelerating gradient in linear accelerators. However, it is desirable to have a constant geometry of the accelerating cells in order to simplify manufacturing. Such geometry leads to a nonsynchronism. A separatrix formalism has been developed for a superconducting linear accelerator based on a model of stationary separatrixes overlapping, and an effective separatrix, moving together with a quasisynchronous particle. This formalism is applied to two cases: either the phase velocity changes from cavity to cavity, or the phase velocity is constant for cavities, belonging to one family. Solving Hamiltonian equations, it is shown qualitatively and quantitatively, how the quasisynchronous phase velocity has to be adjusted either by a stepped phase velocity or by a stepped rf phasing in order to minimize the effect of nonsynchronism in the first or the second case.
\end{abstract}

DOI: $10.1103 /$ PhysRevSTAB.6.124001

\section{INTRODUCTION}

In the beginning superconducting (SC) cavities had a quite simple shape and were operated at relatively low magnetic and electrical fields. Present day advanced technology allows the production of complicated SC cavities with surface electrical and magnetic fields around $60-80 \mathrm{MV} / \mathrm{m}$ and $80-100 \mathrm{mT}$. Because of this the geometry of SC cavities has to be simplified as much as possible to keep costs down, which forbids having a SC cavity for a proton machine whose geometry is changing with acceleration.

A first step of accelerating structure technology simplification had been partly done already in normalconducting linear accelerators in the relative velocity region, $\beta=0.5-0.9$, for meson factories to make them cheaper (Los Alamos National Laboratory, Institute for Nuclear Research in Moscow). Accelerators of this class consist of about 100 cavities, and each cavity has about 20 similar accelerating cells. Obviously, each cavity has a constant phase velocity, and it changes step by step from cavity to cavity. The step value of the phase velocity is determined by the required superposition of neighbor section separatrixes to provide an instantaneous stable motion with minimum deviation from the average phase in the longitudinal plane. Such structures have been named stepped phase velocity structures [1], and their technology can be applied for superconducting linear accelerator as well.

The cavity design in Spallation Neutron Source (SNS), Japan Hadron Factory (JHF), and European Spallation Source (ESS) projects can be considered as a next step of SC cavity simplification, when many cavities belonging to one family have identical geometry [2]. It means the phase velocity changes step by step from family to family. The number of gaps in a cavity and the number of cavities in one family can be varied from one to a few tens, depending on many factors. Of course, such accelerators are simpler and cheaper, but the longitudinal dynamics is more complicated. The particles are sliding
PACS numbers: 29.17.+w, 29.27.Bd, 41.75.-i

down or up relative to the rf wave in dependence of the ratio between the particles and the wave velocities. Thus, the particles are almost never in synchronism with the equivalent traveling wave, and they have no instantaneous stability. However, abandoning synchronism, we acquire freedom in the choice of the rf phase shift, $\Delta \varphi_{\text {str }}$, between relatively short SC cavities. By proper phasing of the rf cavities one can provide a stable quasisynchronous motion in the whole accelerator. This case is called the stepped rf phase structure.

The SC structures of SNS, JHF, and ESS are based on the elliptical SC cavities with very high accelerating gradients $25-30 \mathrm{MeV} / \mathrm{m}$. They are supposed to work in the region of high relative velocity $\beta=0.5-1.0$, when the efficiency of acceleration in the cavity with constant geometry is still enough high. To get higher mechanical rigidity in the region of $\beta=0.1-0.4$ the SC structures are based on the quarter-, half-wave, and $H$-type crossbar resonators (spoke resonators). They have the smaller number of accelerating gaps, but they relate to the stepped rf phase structure as well.

A separatrix formalism for both the stepped phase velocity and the stepped $\mathrm{rf}$ phase structures has been developed. This formalism is especially useful in the design and the tuning of superconducting linear accelerator in the low $\beta$ region, since the technique developed for the higher $\beta$ gives insufficient approximation [2]. The lowest value of $\beta$, the number of gaps in one cavity, the number of cavities in one family, and the rf synchronizing law for cavities are included in the separatrix formalism. In particular, this technique has been used for the design of a superconducting linear accelerator for $H^{-}$and $D^{-}$ ions to serve as an injector into the COSY ring in Jülich providing an energy gain from 2 to $50 \mathrm{MeV}$ [3].

\section{ACCELERATORS WITH STEPPED PHASE VELOCITY STRUCTURE}

At first the stepped phase velocity structure is considered, when $\beta_{\text {str }}$ is constant over the cavity and changes 
from cavity to cavity. Here the quasisynchronous particle and the quasisynchronous phase velocity in both cases will be defined.

The accelerating electrical field, $E(x, y, z) \cos \omega t$, in a periodical structure with period $D$ can be represented through a Fourier expansion:

$$
E(x, y, z, t)=\frac{1}{2} \sum_{n=-\infty}^{\infty} E_{n}(x, y) \cos \left(\omega t-\frac{2 \pi \cdot n}{D} z\right) .
$$

The structure phase velocity is defined as the velocity of a fixed rf phase, when

$$
\begin{gathered}
\omega t-\frac{2 \pi \cdot n}{D} z=\text { const, } \\
\beta_{\text {str }}=\frac{D}{n \lambda} .
\end{gathered}
$$

Further, the traveling wave formalism is used and the fundamental accelerating harmonic is denoted as $E_{\mathrm{ac}}=$ $\frac{1}{2} E_{n}$. Usually, it is the first harmonic $(n=1)$. In a structure with a growing accelerating period $D(z)=\beta_{s}(z) \lambda$ a particle moving with the structural phase velocity $\beta_{s}=$ $\beta_{\text {str }}(t \in 0-\infty)$ is called a synchronous particle and accordingly $\beta_{s}$ is the synchronous phase velocity.

From Eqs. (1) and (2) at any moment $t$, when the particle has a coordinate $z$, the instantaneous phase $\phi$ of the arbitrary particle relative to the synchronous particle phase $\varphi_{s}$ is

$$
\phi=\varphi-\varphi_{s}=\omega \int_{0}^{z} \frac{d \xi}{c \beta(\xi)}-2 \pi \int_{0}^{z} \frac{d \xi}{D(\xi)} .
$$

However, in the stepped geometry structure the period $D_{i}$ changes step by step either from the $i$ th cavity to the $(i+1)$ th cavity, or from the $i$ th family to the $(i+1)$ th family and equals $D_{i}=\beta_{\text {stri }} \lambda$. Obviously, in that case there is no synchronous particle, since all particles oscillate with double frequencies: the eigenfrequency $\Omega$ and external frequency $\nu_{\beta}=\left(2 \pi / T_{D}\right)$,

$$
\varphi=A \cos (\Omega t+\theta)+b \cos \left(\nu_{\beta} t+\vartheta\right)+\varphi_{0},
$$

where $T_{D}$ is the periodicity of $D_{i}$ and $A, b$ are free and forced oscillation amplitudes correspondingly.

The particle, oscillating with external frequency only $(A=0)$, we call the quasisynchronous particle, and its velocity as the quasisynchronous phase velocity.

\section{A. Stationary separatrices crossing and accelerating of quasisynchronous particle}

Since $\beta_{\text {stri }}$ is constant over the cavity, the particle motion in each cavity is described by an equation as in a stationary separatrix with $\varphi_{s}=-90^{\circ}$ :

$$
\frac{d \phi}{d \tau}=\frac{\beta_{\mathrm{str} i}-\beta}{\beta_{\mathrm{str} i}}, \quad \frac{d}{d \tau}\left(\beta_{\mathrm{str} i}-\beta\right)=-A_{E} \sin \phi,
$$

where $A_{E}=\left[\left(e E_{\mathrm{ac}} \lambda\right) /\left(2 \pi m_{0} c^{2} \gamma^{3}\right)\right]$ is a slowly changing constant determined by the acceleration rate, $d \tau=$ $\omega d t=\left(2 \pi / T_{\mathrm{rf}}\right) d t$ is the newly normalized time coordinate, and the phase $\phi$ is counted out from the separatrix center located in the phase $\varphi_{s}=-90^{\circ}$.

Passing from one cavity with $\beta_{\text {stri }}$ into another with bigger $\beta_{\text {stri } i 1}$, the bunch is transferred into a separatrix located higher. Because of a special ratio between $\beta_{\text {str }}$ of neighbor cavities the bunch is always located in one of the stationary separatrices and never outside. The last remark is not met in stepped $\mathrm{rf}$ phase structures. To minimize the bunch coherent oscillation the exit phase of the quasisynchronous particle equals the entrance phase in the next cavity. Thus, all particles oscillate around some average phase $\bar{\phi}_{s}$. In such a structure the particle with the minimum oscillating amplitude is the quasisynchronous particle. Figure 1 shows schematically the phase trajectories of different particles. From Eq. (5) we can derive the oscillation equation for the arbitrary particle in the $i$ th separatrix: $\left(d^{2} \phi / d \tau^{2}\right)+\Omega^{2} \sin \phi=0$, where $\Omega^{2}=$ $\left(A_{E} / \beta_{\text {str }}\right)$ is the longitudinal oscillation frequency in the traveling wave field with phase velocity $\beta_{\text {str. }}$. Since we force all particles to oscillate around some average phase $\bar{\phi}_{s}$, let us expand $\sin \phi$ in the $\bar{\phi}_{s}$ vicinity in terms of $\psi=$ $\phi-\bar{\phi}_{s}+\tan \bar{\phi}_{s}$ :

$$
\frac{d^{2} \psi}{d \tau^{2}}+\Omega^{2} \cos \bar{\phi}_{s} \cdot \psi=0
$$

The velocity and phase deviations upon entrance and exit of the cavity are coupled through the matrix:

$$
\begin{aligned}
{\left[\begin{array}{c}
\psi \\
\beta-\beta_{\text {stri }} \\
\beta_{\text {stri }}
\end{array}\right]_{\text {out }} } & {\left[\begin{array}{cc}
\cos \Phi & -\frac{1}{\Omega \cos ^{1 / 2} \bar{\phi}_{s}} \sin \Phi \\
\Omega \cos ^{1 / 2} \bar{\phi}_{s} \cdot \sin \Phi & \cos \Phi
\end{array}\right] } \\
& \times\left[\begin{array}{c}
\psi \\
\beta-\beta_{\text {stri }} \\
\beta_{\text {stri }}
\end{array}\right]_{0}
\end{aligned}
$$

where $\Phi=\Omega \cos ^{1 / 2} \bar{\phi}_{s} \cdot \pi \cdot n$ is the advanced phase of the longitudinal oscillation per one cavity, and $n$ is the number of periods in a cavity. Since the minimum oscillation is reached at $\psi_{\text {out }}=\psi_{0}$ and

$$
\left.\frac{\beta-\beta_{\text {stri }}}{\beta_{\text {stri }}}\right|_{\text {out }}=-\left.\frac{\beta-\beta_{\text {stri }}}{\beta_{\text {stri }}}\right|_{0},
$$

we can write the expression of the initial phase $\psi_{0}$ and the oscillation amplitude $\Delta \varphi$ for a quasisynchronous particle:

$$
\psi_{0}=\frac{1}{\Omega \cos ^{1 / 2} \bar{\phi}_{s} \tan \frac{\Phi}{2}}\left(\frac{\beta_{\mathrm{qs}}-\beta_{\mathrm{str} i}}{\beta_{\mathrm{stri}}}\right),
$$

and

$$
\Delta \varphi=\frac{1}{\Omega \cos ^{1 / 2} \bar{\phi}_{s}}\left(\frac{\beta_{\mathrm{qs}}-\beta_{\mathrm{str} i}}{\beta_{\text {stri }}}\right)_{\text {out }} \tan \frac{\Phi}{4},
$$

where $\beta_{\mathrm{qs}}$ is the relative velocity of the quasisynchronous particle. 


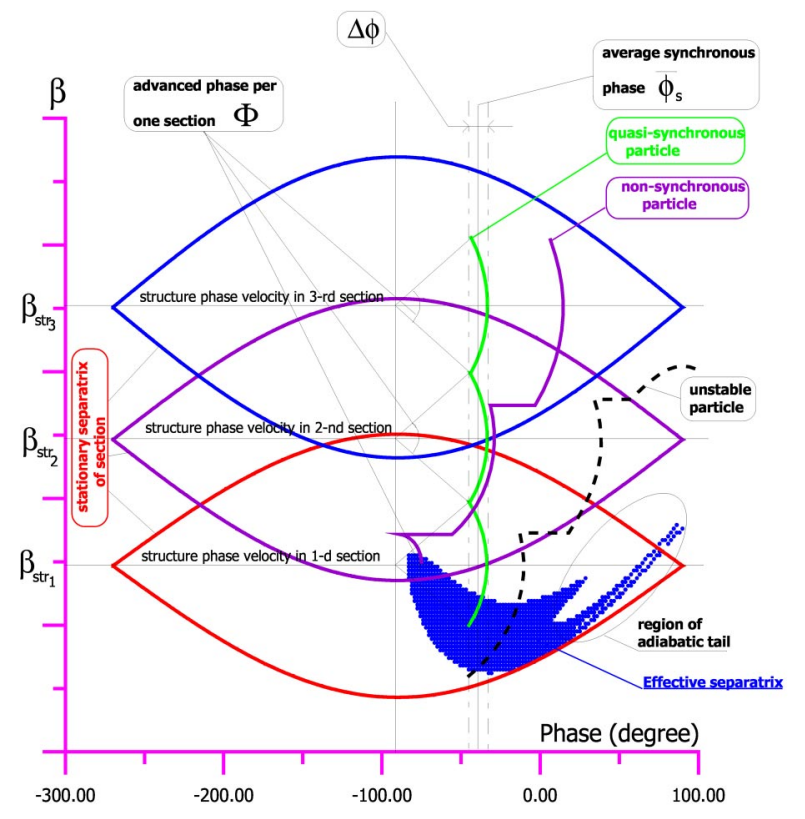

FIG. 1. (Color) Longitudinal motion in the stepped phase velocity structure.

Determining the average phase, as $\bar{\varphi}_{s}=\psi_{0}+\frac{1}{2} \Delta \varphi$, we find the velocity gain $\Delta \beta_{\mathrm{qs}}$ for the quasisynchronous particle over the whole cavity:

$$
\frac{\Delta \beta_{\mathrm{qs}}}{\beta_{\mathrm{stri} i}}=4 \Omega \cos ^{1 / 2} \bar{\phi}_{s} \cdot \tan \bar{\phi}_{s} \cdot \tan \left(\frac{\Omega \cos ^{1 / 2} \bar{\phi}_{s} \cdot \pi n}{4}\right) .
$$

For comparison in accelerators with a gradually growing period of acceleration the velocity gain per $n$ periods is $(\Delta \beta / \beta)=\Omega^{2} \sin \varphi_{s} \cdot \pi n$, and it is similar to Eq. (9) for a small value of $\Omega \pi n$.

\section{B. Effective separatrix creation}

Thus, due to cavity design simplification there are no synchronous particles in the stepped phase velocity structure. The whole bunch always makes a coherent oscillation, and a stable motion relative to a quasisynchronous particle determines the stability of any particle. The above technique is based on a motion description in each stationary separatrix of a cavity and their linking. However, it does not give any information about the effective separatrix, which arises around a quasisynchronous particle and moves together with it. The effective separatrix is the most important characteristic in the longitudinal plane. The effective separatrix is created in the following way. From Eq. (5) we can see the difference between the motion in the structure with a gradually growing period of acceleration and the motion in an accelerator consisting of cavities with $\beta_{\text {stri }}=$ const. In order to make them absolutely similar the structure phase velocity of accelerating cavities has to change in accordance with the law, where one would add in a second equation of (5) some constant $\left(d \beta_{\text {str }} / d \tau\right)=\alpha$ :

$$
\frac{d \phi}{d \tau}=\frac{\beta_{\text {stri }}-\beta}{\beta_{\text {stri }}}, \quad \frac{d}{d \tau}\left(\beta_{\text {stri }}-\beta\right)=-A_{E} \sin \phi+\alpha,
$$

where $A_{E}$ is determined by Eq. (5).

Obviously, if one takes $\alpha=A_{E} \sin \bar{\phi}_{s}$ all particles oscillate around the phase

$$
\bar{\phi}_{s}=\arcsin \left(\frac{\alpha}{A_{E}}\right)
$$

and one reaches full identity with a gradually growing period structure. Of course, this is not a realistic case, since in practice $\beta_{\text {stri }}$ is the stepwise function with step value $\delta \beta=\beta_{\text {stri } i+1}-\beta_{\text {stri }}$ (see Fig. 2). It can be represented through the linearly growing component $\bar{\beta}_{\text {str }}=$ $\alpha \tau$ and the sawtooth oscillating component $\tilde{\beta}_{\text {str }}$, which can be expanded in a Fourier series. As a result one has

$$
\begin{aligned}
\beta_{\mathrm{str}}(\tau) & =\bar{\beta}_{\mathrm{str}}(\tau)+\tilde{\beta}_{\mathrm{str}}(\tau) \\
& =\alpha \tau+\delta \beta\left(\frac{1}{2}+\sum_{m=1}^{\infty} \frac{1}{\pi m} \sin m \nu_{\beta} \tau\right),
\end{aligned}
$$

where $\nu_{\beta}=\frac{1}{n}$ is the dimensionless frequency, and $T=$ $1 / \nu_{\beta}=n$ is the periodicity of the phase-stepped velocity function. Thus, taking into account the stepwise character

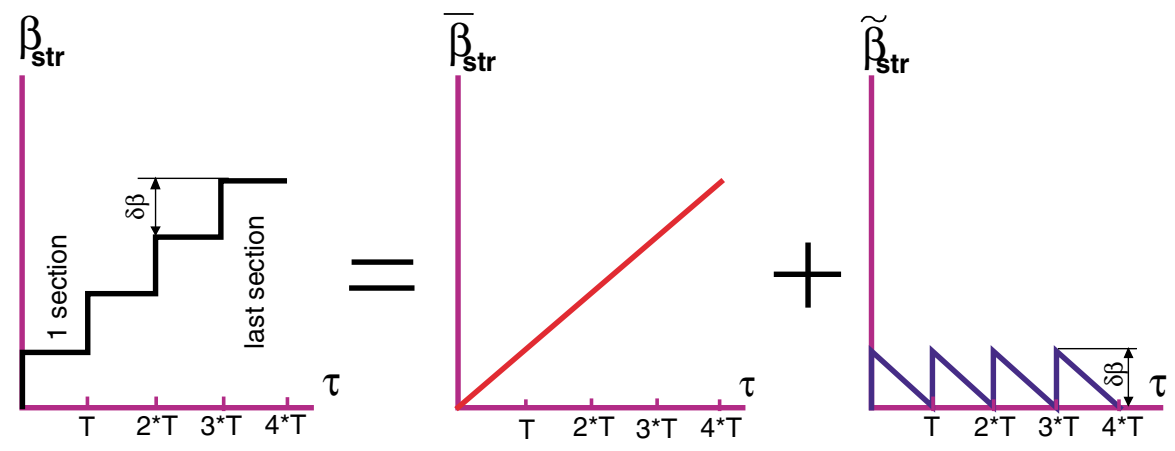

FIG. 2. (Color) Representation of velocity function in the stepped phase velocity structure. 
of $\beta_{\text {str }}$, it is reasonable to rewrite Eq. (10) as

$$
\begin{aligned}
\frac{d \phi}{d \tau} & =1-\frac{\beta}{\beta_{\mathrm{str}}}, \\
\frac{d}{d \tau}\left(\beta_{\mathrm{str}}-\beta\right) & =\alpha+\frac{\delta \beta \nu_{\beta}}{\pi} \sum_{m=1}^{\infty} \cos m \nu_{\beta} \tau-A_{E} \sin \phi .
\end{aligned}
$$

Differentiating the first equation of (13) and substituting the second equation, one has

$$
\frac{d^{2} \phi}{d \tau^{2}}+\frac{A_{E}}{\beta_{\mathrm{str}}} \sin \phi-\frac{\alpha}{\beta_{\mathrm{str}}}-\frac{\delta \beta}{\beta_{\mathrm{str}}} \cdot \frac{\nu_{\beta}}{\pi} \sum_{m=1}^{\infty} \cos m \nu_{\beta} \tau=0 .
$$

To simplify the term $(d \phi / d \tau)$ is omitted, which is responsible for the Lorentz friction and gives in the solution the factor $\phi \propto e^{-(1 / 2)(d \ln \beta / d \tau) \tau}$. Taking into account (11), the constant term in (14) can be written through the longitudinal frequency:

$$
\frac{\alpha}{\beta_{\mathrm{str}}}=\frac{A_{E}}{\beta_{\mathrm{str}}} \sin \bar{\phi}_{s}, \quad \frac{\alpha}{\beta_{\mathrm{str}}}=\Omega^{2} \sin \bar{\phi}_{s} .
$$

Following an obvious relation $\left(d \bar{\beta}_{\text {str }} / d \tau\right) \cdot 2 \pi \cdot T=$ $\delta \beta$, or $\delta \beta=\left(2 \pi / \nu_{\beta}\right) \alpha$, which means $\left(\delta \beta / \beta_{\text {str }}\right)$. $\left(\nu_{\beta} / \pi\right)=2 \Omega^{2} \sin \bar{\phi}_{s}$, Eq. (14) is represented in the form:

$$
\frac{d^{2} \phi}{d \tau^{2}}+\Omega^{2} \sin \phi-\Omega^{2} \sin \bar{\phi}_{s}=2 \Omega^{2} \sin \bar{\phi}_{s} \sum_{m=1}^{\infty} \cos m \nu_{\beta} \tau \text {. }
$$

For a small deviation $\phi$ from $\bar{\phi}_{s}$ the solution of Eq. (16) is

$$
\begin{aligned}
\phi(\tau)= & A \cos \left(\Omega \cos ^{1 / 2} \bar{\phi}_{s} \cdot \tau+\chi\right)+\bar{\phi}_{s} \\
& +2 \Omega^{2} \sin \bar{\phi}_{s} \cdot \sum_{m=1}^{\infty} \frac{\cos m \nu_{\beta} \tau}{\Omega^{2} \cos \bar{\phi}_{s}-m^{2} \nu_{\beta}^{2}} .
\end{aligned}
$$

From Eq. (17) one can see that all particles oscillate with frequency $\Omega \cos ^{1 / 2} \bar{\phi}_{s}$ around a quasisynchronous particle $\phi_{\mathrm{qs}}$, which itself oscillates around the average synchronous phase $\bar{\phi}_{s}$,

$$
\phi_{\mathrm{qs}}=\bar{\phi}_{s}+2 \Omega^{2} \sin \bar{\phi}_{s} \cdot \sum_{m=1}^{\infty} \frac{\cos m \nu_{\beta}}{\Omega^{2} \cos \bar{\phi}_{s}-m^{2} \nu_{\beta}^{2}} \tau
$$

with the frequency spectrum $\nu_{\beta} \cdot(1-m)$.
Thus, no particle has zero amplitude of oscillation. Since one is interested in the stable region relative to the oscillating quasisynchronous particle, one uses a new coordinate system that is moving together with a quasisynchronous particle $\psi=\phi-\bar{\phi}_{s}-f(\tau)$, where

$$
f(\tau)=2 \Omega^{2} \sin \bar{\phi}_{s} \sum_{m=1}^{\infty} \frac{\cos m \nu_{\beta} \tau}{\Omega^{2} \cos \bar{\phi}_{s}-m^{2} \nu_{\beta}^{2}} .
$$

The solution of Eq. (16) depends on the ratio between the perturbation frequency $\nu_{\beta}$ and the longitudinal frequency $\Omega \cos ^{1 / 2} \bar{\phi}_{s}$.

In case $\nu_{\beta} \gg \Omega \cos ^{1 / 2} \bar{\phi}_{s}$, it is the motion in a fast oscillating field, and one retains the lowest harmonic only $f(\tau)=\varphi_{a} \cos \nu_{\beta} \tau$ with $\varphi_{a}=\left(2 \Omega^{2} \sin \bar{\phi}_{s} / \nu_{\beta}^{2}\right)$.

Obviously, the quasisynchronous particle moving along the trajectory $\bar{\phi}_{s}+f(\tau)$ does not experience the action of external forces and in the new coordinates $\left\{\Delta \beta=\beta_{\mathrm{qs}}-\beta ; \psi=\phi-\phi_{\mathrm{qs}}\right\}$ one has the equations:

$$
\begin{aligned}
\frac{d \psi}{d \tau}=\frac{\Delta \beta}{\beta_{\mathrm{qs}}}, & \\
\frac{d(\Delta \beta)}{d \tau}=-A_{E} \cdot & {\left[\cos \left(\psi+\bar{\phi}_{s}+\varphi_{a} \cos \nu_{\beta} \tau\right)\right.} \\
& \left.-\cos \left(\bar{\phi}_{s}+\varphi_{a} \cos \nu_{\beta} \tau\right)\right] .
\end{aligned}
$$

In Eq. (18) the new $\phi_{s}$ was changed by $90^{\circ}-\phi_{s}$ and passed in the "cos"-wave coordinate system just to make it similar to the generally accepted designation. So, one has the explicit time-dependent Hamiltonian:

$$
H(\Delta \beta, \psi, \tau)=\frac{(\Delta \beta)^{2}}{2 \beta_{q s}}+U(\psi)
$$

with the potential energy:

$$
\begin{aligned}
U(\psi)=A_{E} \cdot[ & \sin \left(\psi+\bar{\phi}_{s}+\varphi_{a} \cos \nu_{\beta} \tau\right) \\
& \left.-\psi \cos \left(\bar{\phi}_{s}+\varphi_{a} \cos \nu_{\beta} \tau\right)\right] .
\end{aligned}
$$

Taking into account the expansions

$$
\begin{aligned}
\cos \left(\cos \nu_{\beta} \tau\right) & =J_{0}\left(\varphi_{a}\right)+2 J_{2}\left(\varphi_{a}\right) \cos 2 \nu_{\beta} \tau+\cdots, \\
\sin \left(\cos \nu_{\beta} \tau\right) & =2 J_{1}\left(\varphi_{a}\right) \cos \nu_{\beta} \tau-2 J_{3}\left(\varphi_{a}\right) \cos 3 \nu_{\beta} \tau+\cdots,
\end{aligned}
$$

where $J_{n}\left(\varphi_{a}\right)$ is the Bessel function of the $n$th order, and retaining a reasonable number of terms, the force $d(\Delta \beta) / d \tau$ is represented as

$$
\begin{aligned}
\frac{d(\Delta \beta)}{d \tau}= & -A_{E} \cdot J_{0}\left(\varphi_{a}\right) \cdot\left[\cos \left(\psi+\bar{\phi}_{s}\right)-\cos \bar{\phi}_{s}\right] \\
& +A_{E} \cdot 2 J_{1}\left(\varphi_{a}\right) \cdot\left[\sin \left(\psi+\phi_{s}\right)-\sin \bar{\phi}_{s}\right] \cdot \cos \nu_{\beta} \tau-A_{E} \cdot 2 J_{2}\left(\varphi_{a}\right)\left[\cos \left(\psi+\bar{\phi}_{s}\right)-\cos \bar{\phi}_{s}\right] \cdot \cos 2 \nu_{\beta} \tau
\end{aligned}
$$

The action of the fast oscillating component can be found by the Landau method [4], when the effective potential is represented through the sum of a time-independent averaged fast oscillating component: 


$$
\frac{d U_{\mathrm{eff}}}{d \phi}=\frac{d U}{d \phi}+\overline{\frac{d p}{d \tau} \frac{\partial}{\partial \phi}\left(\frac{d p}{d \tau}\right)} .
$$

As a results one gets

$$
\begin{aligned}
H(\Delta \beta, \psi)= & \frac{(\Delta \beta)^{2}}{2 \beta_{q s}}+A_{E} \cdot J_{0}\left(\varphi_{a}\right) \\
& \cdot\left[\sin \left(\psi+\bar{\phi}_{s}\right)-\psi \cos \bar{\phi}_{s}\right]+A_{E}^{2} \cdot J_{1}^{2}\left(\varphi_{a}\right) \cdot \sin ^{2}\left(\psi+\bar{\phi}_{s}\right)-A_{E}^{2} \cdot J_{2}^{2}\left(\varphi_{a}\right) \cdot \cos ^{2}\left(\psi+\bar{\phi}_{s}\right) .
\end{aligned}
$$

Hamiltonian (24) gives a full description of the system and allows one to find the effective separatrix. Now one can conclude that in the stepped phase velocity structure due to coherent oscillation of the whole separatrix with the frequency of a quasisynchronous particle and an amplitude $\varphi_{a}$, the effective longitudinal frequency decreases proportionally to $\sqrt{J_{0}\left(\varphi_{a}\right)}$, and consequently the velocity spread of the separatrix decreases by the same factor. Since another two terms $A_{E}^{2}\left(\varphi_{a}^{2} / 4\right)$ and $A_{E}^{2}\left(\varphi_{a}^{4} / 64\right)$ are negligible, the phase length of separatrix remains the same. The first term $\sqrt{J_{0}\left(\varphi_{a}\right)}$ expansion is $1-\left(\varphi_{a}^{2} / 8\right)$; that is, for a relatively large oscillation of the quasisynchronous particle the separatrix changes insignificantly. For instance, for the $\pm 30^{\circ}$ oscillation the separatrix velocity spread decreases by $3 \%-4 \%$ only.

Therefore, the number of cavities in accelerators with a fixed final energy can be decreased as much as one satisfying the condition of beam matching with a separatrix.

Nevertheless, one is restricted by another requirement to avoid an external resonance. For longer cavities the frequency perturbation $\nu_{\beta}$ decreases and it could be comparable (but not equal) to the eigenfrequency $\nu_{\beta} \approx$ $k \Omega \cos ^{1 / 2} \bar{\phi}_{s}(k \geq 2)$. In that case one has an external resonance in the nonlinear system. In first approximation Eq. (16) can be written in the form:

$$
\frac{d^{2} \phi}{d \tau^{2}}+\Omega^{2} \sin \phi=F(\phi, \tau)
$$

where

$$
\begin{aligned}
F(\phi, \tau)= & \Omega^{2} \sin \bar{\phi}_{s}\left[1+2 \sum_{n=1}^{\infty} \cos \nu_{\beta} n \tau\right] \\
& -\Omega^{2} \sin \bar{\phi}_{s}\left[1+2 \sum_{n=1}^{\infty} \cos \nu_{\beta} n \tau\right] \cdot \frac{d \phi}{d \tau} .
\end{aligned}
$$

Now we do not omit the Lorentz damping term, since it can make a significant contribution in the final solution.

Following Bogolyubov's asymptotic method [5], the first approximation solution is sought in the form of

$$
\phi=a(\tau) \cos \psi(\tau), \quad \frac{d \phi}{d \tau}=-a(\tau) \Omega \sin \psi(\tau),
$$

where $\psi(\tau)=\Omega \tau+\theta(\tau)$ and it is determined by the equation system:

$$
\begin{aligned}
& \frac{d a}{d \tau}=\Delta_{t}\left\{-\frac{1}{\Omega} F(-a \Omega \sin \psi, \tau) \sin \psi\right\}, \\
& \frac{d \theta}{d \tau}=\Delta_{t}\left\{-\frac{1}{a \Omega} F(-a \Omega \sin \psi, \tau) \cos \psi\right\},
\end{aligned}
$$

where $\Delta_{t}$ is the operator of averaging. Substituting Eq. (26) into Eq. (28), one has

$$
\begin{aligned}
& \frac{d a}{d \tau}=-a \frac{\Omega^{2} \sin \bar{\phi}_{s}}{2}+a \frac{\Omega^{2} \sin \bar{\phi}_{s}}{2} \frac{\cos \left[\left(2 \Omega-n \nu_{\beta}\right) \tau+\theta\right]}{d \theta}, \\
& \frac{d \theta}{d \tau}=2 \Omega-n \nu_{\beta} .
\end{aligned}
$$

From this equation one sees that in the case of $2 \Omega=\nu_{\beta}$ the solution of this equation can be written

$$
a \propto e^{-\left(\Omega^{2} \sin \bar{\phi}_{s} / 2\right) \tau} \cdot e^{\cos \theta\left(\Omega_{2} \sin \bar{\phi}_{s} / 2\right) \tau} .
$$

The first term is just a Lorentz damping factor existing in any accelerator. However, due to the parametrization of the friction force one has the additional term, which depends on phase $\theta$. For some phase $\theta$ this factor causes additional damping, for another it makes the Lorentz factor weaker. It causes particle losses especially in the region of the adiabatic tail (see Fig. 1). Therefore, in practice one has to make a minimum ratio $\left(\nu_{\beta} / \Omega\right) \approx$ $4-5$, which avoids such phenomena.

\section{ACCELERATORS WITH STEPPED RF PHASE STRUCTURE}

Consider the case, where the cavities are joint in families, and all $n$ cavities of one family have the same structure phase velocity $\beta_{\text {str }}=$ const for $i \in 1-n$. On the one hand, such a linear accelerator is cheaper, since it has a smaller number of cavity types, and all cavities belonging to one family are interchangeable. On the other hand, the violation of synchronism between particles and structure requires another mechanism of acceleration. It will be shown that such a mechanism is based on a special law of rf phase shift from cavity to cavity.

\section{A. The rf phase shift as acceleration mechanism in a structure with one stationary separatrix}

As in the previous case the particles move in a cavity, where the structure phase velocity $\beta_{\text {str }}$ is constant. Therefore, they oscillate around $\varphi_{s}=-90^{\circ}$. But in the considered case the particle velocity deviation from the structure phase velocity $\Delta \beta=\beta_{\text {str }}-\beta$ can exceed the velocity spread of stationary separatrix with a synchronous level $\beta=\beta_{\text {str }}$. The longitudinal phase plane is divided into three regions. Particles higher or lower with respect to the separatrix drift along phase trajectories to the left or the right directions correspondingly (regions III 
and I), and only in region II do the particles oscillate in the separatrix.

Figure 3 explains the mechanism of acceleration. It is based on an rf shift for each cavity. The particles have to return back each time after passing through a cavity to get an average phase $\bar{\phi}_{s}$ over all cavities. By a proper choice of the rf phase shift $\Delta \varphi_{\mathrm{rf}}$ between cavities one can create a quasisynchronous motion and in total a stable motion in the whole accelerator. The quasisynchronous particle oscillating in a cavity around $\varphi=0$ (for the "sin" wave) is forced by the intercavity rf shift to oscillate around $\bar{\phi}_{s}$. The equations of oscillation are similar to a previous case (5), but with one difference: for each cavity the initial and final phase deviations are not equal each to other.

Taking into account the average phase $\bar{\phi}_{s}$ of oscillation, one can write the expressions for initial phase deviation $\varphi_{0}$ and oscillation amplitude $\Delta \varphi$ for a cavity:

$$
\begin{aligned}
\varphi_{0} & =\frac{2 \bar{\phi}_{s}}{1+\cos \Phi}+\frac{\beta-\beta_{\mathrm{str}}}{\beta_{\mathrm{str}}} \cdot \frac{1}{\Omega \cos ^{1 / 2} \bar{\phi}_{s}} \cdot \frac{\sin \Phi}{1+\cos \Phi}, \\
\Delta \varphi & =-\bar{\phi}_{s} \cdot \tan ^{2} \frac{\Phi}{2}-\frac{\beta-\beta_{\mathrm{str}}}{\beta_{\mathrm{str}}} \cdot \frac{2}{\Omega \cos ^{1 / 2} \bar{\phi}_{s}} \cdot \tan \frac{\Phi}{2}
\end{aligned}
$$

where $\Phi=\Omega \cos ^{1 / 2} \bar{\phi}_{s} \pi n$ is the phase advance of the longitudinal oscillation per cavity, and $n$ is the number of gaps in the cavity. If the required velocity gain per cavity is $\Delta \beta$, the average phase of oscillation $\bar{\phi}_{s}$ is derived from the simple formula $\left(\Delta \beta / \beta_{\text {str }}\right)=$ $2 \Omega \sin \bar{\phi}_{s} \tan (\Phi / 2)$, following from Eq. (30).

Since the advanced phase $\Phi$ is a small value, the oscillation amplitude can be defined as

$$
\Delta \varphi=-\frac{\beta-\beta_{\text {str }}}{\beta_{\text {str }}} \cdot \frac{2}{\Omega \cos ^{1 / 2} \bar{\phi}_{s}} \cdot \tan \frac{\Phi}{2} .
$$

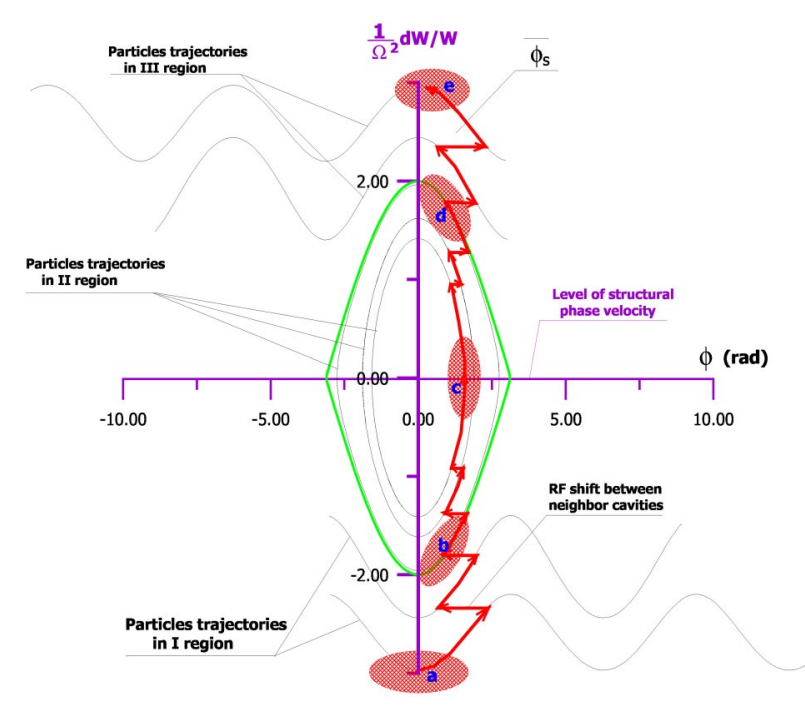

FIG. 3. (Color) Longitudinal motion in the stepped rf phase structure.
In comparison with the stepped phase velocity structure this value [compare with Eq. (8)] is different for each cavity and is much larger due to the bigger velocity deviation.

\section{B. Effective separatrix creation}

Thus, the quasisynchronous particle executes an oscillation with a large amplitude, and, in principle, it is instantaneously unstable nearly all the time. The separatrix in the case of the stepped rf phase is created in the following way. The structure under consideration has a longitudinal periodicity integer of $\beta_{\text {str }} \lambda$. Therefore, one uses the normalized longitudinal coordinate $d \varsigma=$ $2 \pi\left(d z / \beta_{\text {str }} \lambda\right)$, but not as in the previous case with the normalized time $d \tau=2 \pi\left(d t / T_{\mathrm{rf}}\right)$ :

$$
\frac{d \phi}{d \varsigma}=\frac{\beta_{\mathrm{str}}}{\beta}-1, \quad \frac{d \beta}{d \varsigma}=\frac{e E_{\mathrm{ac}} \lambda \beta_{\mathrm{str}}}{2 \pi m_{0} c^{2} \gamma^{3} \beta} \sin \phi
$$

From Eq. (31) one derives the phase oscillation equation $\left(d^{2} \phi / d s^{2}\right)+\Omega^{2} \sin \phi=0$, where $\Omega^{2}=A_{E} \cdot\left(\beta_{\text {str }}^{2} / \beta^{3}\right)$ is determined by means of the parameter $A_{E}=$ $\left[\left(e E_{\mathrm{ac}} \lambda\right) /\left(2 \pi m_{0} c^{2} \gamma^{3}\right)\right]$ introduced before in (5). Obviously, if one does not undertake some action with phase $\phi$, the particles will accelerate around phase $\phi=$ 0 , and acceleration will be absent, since $\Delta W \propto \overline{\sin \phi}_{s}$. To correct this situation one adds the external phase shift $\varphi_{\text {str }}(\mathrm{s})$ to the phase $\phi$. Following definition (3) the corrected phase is

$$
\phi(\mathrm{s})=\int_{0}^{\mathrm{s}} \frac{\beta_{\mathrm{str}} d \xi}{\beta}-\int_{0}^{\mathrm{s}} d \xi+\varphi_{\mathrm{str}}(\mathrm{s})
$$

and the first equation of system (31) takes the form:

$$
\frac{d \phi}{d \varsigma}=\frac{\beta_{\mathrm{str}}}{\beta}-1+\frac{d \varphi_{\mathrm{str}}(\varsigma)}{d \varsigma} .
$$

Then the phase oscillation equation is

$$
\frac{d^{2} \phi}{d \varsigma^{2}}+\Omega^{2} \sin \phi-\frac{d^{2} \varphi_{\text {str }}}{d \varsigma^{2}}=0
$$

In case $\left(d^{2} \varphi_{\text {str }} / d \varsigma^{2}\right)=$ const and not equal to zero, all particles will oscillate around some phase $\bar{\phi}_{s}=$ $\arcsin \left[\left(d^{2} \varphi_{\text {str }} / d \varsigma^{2}\right) / \Omega^{2}\right]$. Thus, the second derivative of $\varphi_{\text {str }}(\varsigma)$ defines the acceleration rate in the stepped rf phase structure.

A stepped $\mathrm{rf}$ phase structure similar to the structure with a gradually growing period of acceleration can be found. Since the rf shift is coherent and acts on the whole bunch, it is reasonable to find a function $\varphi_{\text {str }}(\varsigma)$ from the synchronous particle condition: 


$$
\begin{aligned}
& \frac{d \bar{\phi}_{s}}{d \varsigma}=0 \Rightarrow \frac{d \varphi_{\mathrm{str}}(\mathrm{s})}{d \varsigma}=1-\frac{\beta_{\mathrm{str}}}{\beta_{\mathrm{s}}}, \\
& \frac{d \beta_{s}}{d \varsigma}=A_{E} \frac{\beta_{\mathrm{str}}}{\beta_{s}} \sin \bar{\phi}_{s} .
\end{aligned}
$$

Differentiating the first and substituting the second equation there, one gets an equation for $\varphi_{\text {str }}(\mathrm{s})$ :

$$
\frac{d^{2} \varphi_{\mathrm{str}}(\varsigma)}{d \varsigma^{2}}=A_{E} \cdot \frac{\beta_{\mathrm{str}}^{2}}{\beta_{s}^{3}} \sin \bar{\phi}_{s}
$$

Substituting Eq. (36) into Eq. (34) one can conclude: realizing a structure with an $\mathrm{rf}$ shift according to Eq. (36), one has a structure similar to the structure with a gradually growing period of acceleration with synchronous phase $\bar{\phi}_{s}$. It is easy to find the function for $\varphi_{\text {str }}(\varsigma)$. From the second equation (35) one gets

$$
\frac{\beta_{s}^{2}}{2}-\frac{\beta_{s 0}^{2}}{2}=A_{E} \sin \bar{\phi}_{s} \cdot \beta_{\mathrm{str}} \cdot \mathrm{s} .
$$

Substituting (37) into (36), one gets

$$
\frac{d^{2} \varphi_{\mathrm{str}}}{d \varsigma^{2}}=\frac{A_{E} \sin \bar{\phi}_{s} \cdot \beta_{\mathrm{str}}^{2}}{\left(2 A_{E} \sin \bar{\phi}_{s} \cdot \beta_{\mathrm{str}} \varsigma+\beta_{s 0}^{2}\right)^{3 / 2}} .
$$

Double integrating Eq. (38) and taking into account $\varphi_{\text {str }}(0)=0$ and

$$
\left.\frac{d \varphi_{\mathrm{str}}}{d \mathrm{~s}}\right|_{\beta=\beta_{\mathrm{str}}}=0
$$

one gets the full definition of $\varphi_{\text {str }}(\varsigma)$ :

$$
\begin{aligned}
\frac{d \varphi_{\mathrm{str}}(\mathrm{s})}{d \mathrm{~s}}= & 1-\frac{\beta_{\mathrm{str}}}{\left(2 A_{E} \sin \bar{\phi}_{s} \cdot \beta_{\mathrm{str}} \cdot \varsigma+\beta_{s 0}^{2}\right)^{1 / 2}}, \\
\varphi_{\mathrm{str}}(\mathrm{\varsigma})= & -\frac{1}{A_{\beta}}\left(2 A_{E} \sin \bar{\phi}_{s} \cdot \beta_{\mathrm{str}} \cdot \varsigma+\beta_{s 0}^{2}\right)^{1 / 2}+\varsigma \\
& +\frac{\beta_{s 0}}{\mathrm{~A}_{E} \sin \bar{\phi}_{s}} .
\end{aligned}
$$

This determines the rf phase shift function, which one ideally provides for acceleration in a one phase velocity structure.

However, such a function could be realized only in the case when the rf phase of the cavity is shifted together with the sliding particle [first equation of (31)]. In reality the rf phase of each cavity is fixed and changes from cavity to cavity step by step. The step value is proportional to periodicity $T$ of the cavities. Therefore, the closest approach to the ideal case is the cavity with one gap. However, for economical reasons one would like to have a longer cavity with some gaps. Figure 4 shows the function $\varphi_{\text {str }}(s)$, which one could realize in practice (left picture). It is a stepwise function with an average value $\bar{\varphi}_{\text {str }}$ coinciding with an rf shift of the ideal case (38) and (39). Introducing, like in the previous case, a "triangular" function $\tilde{\varphi}_{\text {norm }}=\sum_{m=1}^{\infty}(1 / \pi m) \sin m \nu_{\mathrm{ph}} \varsigma$, the real $\varphi_{\text {str }}(\varsigma)$ could be submitted through the sum:

$$
\varphi_{\mathrm{str}}(\mathrm{\varsigma})=\bar{\varphi}_{\mathrm{str}}(\mathrm{\varsigma})+\frac{d \bar{\varphi}_{\mathrm{str}}}{d \varsigma} \cdot 2 \pi \cdot T \cdot \tilde{\varphi}_{\mathrm{norm}}(\mathrm{s})
$$

where $T$ is the period of cavity repetition and equals numerically the number of gaps in one cavity.

As distinct from the stepped phase velocity structure in the considered structure the amplitude of triangular function is proportional to the derivative $\left(d \bar{\varphi}_{\text {str }} / d \varsigma\right) \cdot 2 \pi \cdot T$ :

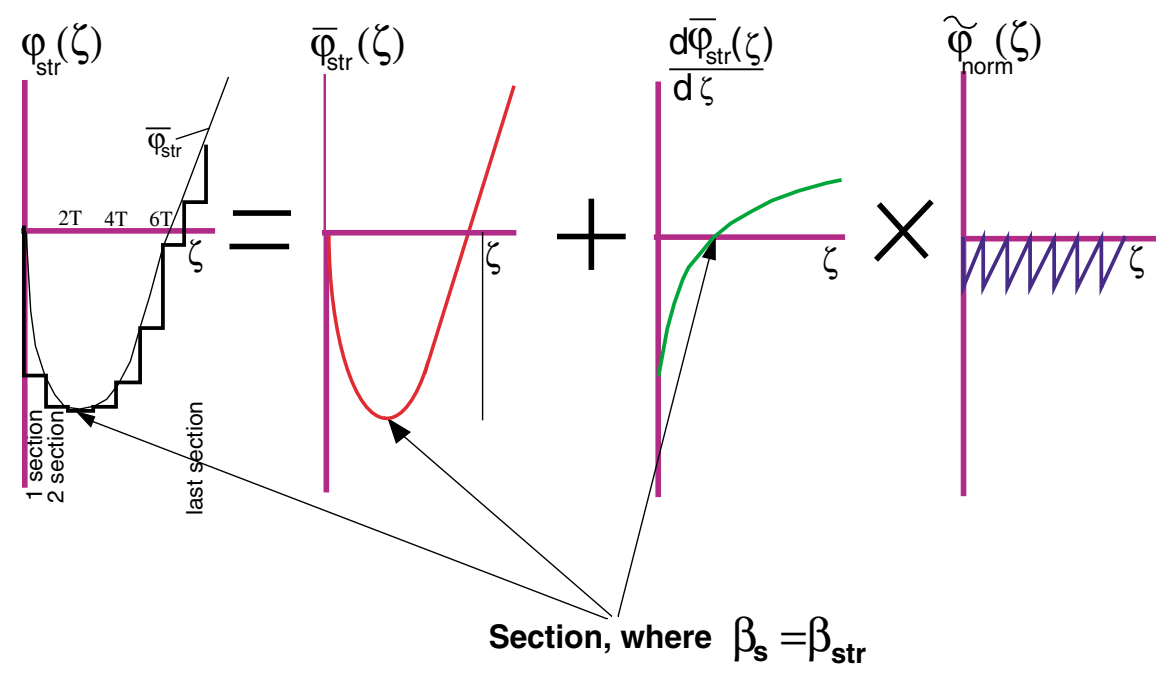

FIG. 4. (Color) Intersection rf phasing in structure. 


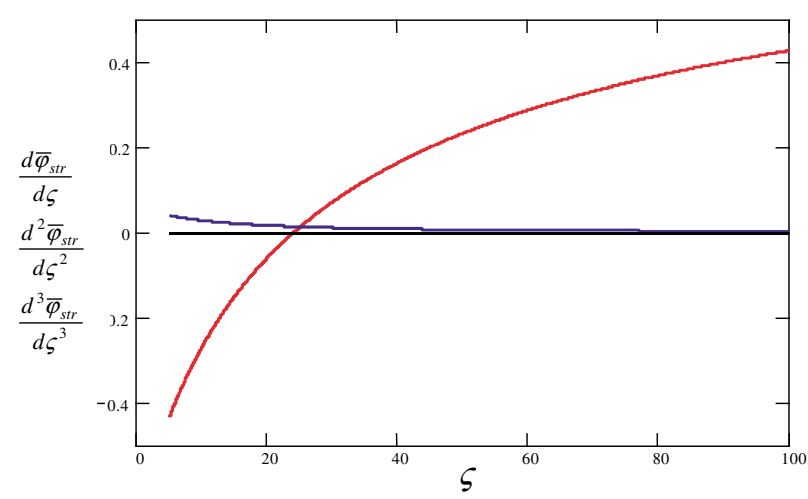

FIG. 5. (Color) Dependence of first (red line), second (blue line), and third (black line) derivatives versus normalized time.

$$
\tilde{\varphi}_{\mathrm{str}}=\frac{d \bar{\varphi}_{\mathrm{str}}}{d \varsigma} \cdot 2 \pi \cdot T \cdot \sum_{m=1}^{\infty} \frac{1}{\pi m} \sin m \nu_{\mathrm{ph}} \varsigma,
$$

where $\nu_{\mathrm{ph}}=(1 / T)$.

Substituting Eqs. (40) and (41) into (34) and taking into account (36), one gets

$$
\begin{aligned}
\frac{d^{2} \phi}{d \varsigma^{2}}+\Omega^{2} \sin \phi-\Omega^{2} \sin \bar{\phi}_{s}= & 2 \frac{d^{3} \bar{\varphi}_{\mathrm{str}}}{d \varsigma^{3}} \sum_{m=1}^{\infty} \frac{\sin m \nu_{\mathrm{ph}} \varsigma}{m \nu_{\mathrm{ph}}} \\
& +4 \frac{d^{2} \bar{\varphi}_{\mathrm{str}}}{d \varsigma^{2}} \sum_{m=1}^{\infty} \cos m \nu_{\mathrm{ph}} \varsigma \\
& -2 \frac{d \bar{\varphi}_{\mathrm{str}}}{d \varsigma} \sum_{m=1}^{\infty} m \nu_{\mathrm{ph}} \sin m \nu_{\mathrm{ph}} \varsigma
\end{aligned}
$$

To compare the contribution of the terms on the right side of (43) all derivatives are written in more explicit form using the current velocity $\beta$ :

$$
\begin{aligned}
\frac{d \bar{\varphi}_{\mathrm{str}}}{d \varsigma} & =1-\frac{\beta_{\mathrm{str}}}{\beta(\mathrm{s})} ; \quad \frac{d^{2} \bar{\varphi}_{\mathrm{str}}}{d \varsigma^{2}}=\Omega^{2}(\mathrm{~s}) \sin \bar{\phi}_{s}, \\
\frac{d^{3} \bar{\varphi}_{\mathrm{str}}}{d \varsigma^{3}} & =3 \frac{\beta(\varsigma)}{\beta_{\mathrm{str}}} \Omega^{4} \sin ^{2} \bar{\phi}_{s} .
\end{aligned}
$$

Figure 5 shows the behavior of the derivatives along the accelerator. One can see that the contribution of the second and third derivatives on the right side is negligible. All derivatives are slowly varying functions, therefore, the first derivative can be considered as a slowly timedependent coefficient.
In the vicinity of $\bar{\phi}_{s}$ one can write for Eq. (42):

$$
\begin{aligned}
\phi(\mathrm{s})= & A \cos \left(\Omega \cos ^{1 / 2} \bar{\phi}_{s} \cdot \mathrm{s}+\chi\right)+\bar{\phi}_{s} \\
& -2 \frac{d \bar{\varphi}_{\mathrm{str}}}{d \varsigma} \cdot \sum_{m=1}^{\infty} \frac{m \nu_{\mathrm{ph}} \cdot \sin m \nu_{\mathrm{ph}} \varsigma}{\Omega^{2} \cos \bar{\phi}_{s}-m^{2} \nu_{\mathrm{ph}}^{2}}
\end{aligned}
$$

As in the case of the stepped phase velocity one defines the particle oscillating only with external frequency as a quasisynchronous particle:

$$
\phi_{\mathrm{qs}}=\bar{\phi}_{s}-2 \frac{d \bar{\varphi}_{\mathrm{str}}}{d \varsigma} \cdot \sum_{m=1}^{\infty} \frac{m \nu_{\mathrm{ph}} \cdot \sin m \nu_{\mathrm{ph}} \varsigma}{\Omega^{2} \cos \bar{\phi}_{s}-m^{2} \nu_{\mathrm{ph}}^{2}} .
$$

Applying a coordinate system moving together with the quasisynchronous particle $\psi=\phi-\bar{\phi}_{s}-f(\varsigma)$, where $f(\mathbf{s})$ is

$$
f(\varsigma)=-2 \frac{d \bar{\varphi}_{\mathrm{str}}}{d \varsigma} \cdot \sum_{m=1}^{\infty} \frac{m \nu_{\mathrm{ph}} \cdot \sin m \nu_{\mathrm{ph}} \varsigma}{\Omega^{2} \cos \bar{\phi}_{s}-m^{2} \nu_{\mathrm{ph}}^{2}} .
$$

For $\nu_{\beta} \gg \Omega \cos ^{1 / 2} \bar{\phi}_{s}$ one retains the first harmonic only $f(\mathrm{~s})=\varphi_{a} \sin \nu_{\mathrm{ph}} \varsigma$ with $\varphi_{a}=\left(2 / \nu_{\mathrm{ph}}\right) \cdot\left(d \bar{\varphi}_{\text {str }} / d \varsigma\right)$.

In the cos wave and using new coordinates $\{\Delta \beta=$ $\left.\beta_{\mathrm{qs}}-\beta ; \psi=\phi-\bar{\phi}_{s}-f(\mathrm{~s})\right\}$ one gets the equation system:

$$
\begin{aligned}
\frac{d \psi}{d \varsigma}=\frac{\beta_{\mathrm{str}}}{\beta_{\mathrm{qs}}^{2}} \cdot \Delta \beta, & \\
\frac{d \Delta \beta}{d \varsigma}=-A_{E} \frac{\beta_{\mathrm{str}}}{\beta_{\mathrm{qs}}} & \cos \left(\psi+\bar{\phi}_{s}+\varphi_{a} \sin \nu_{\mathrm{ph} \varsigma)}\right. \\
& \left.-\cos \left(\bar{\phi}_{s}+\varphi_{a} \sin \nu_{\mathrm{ph}} \varsigma\right)\right] .
\end{aligned}
$$

Thus one obtains the explicit time-dependent Hamiltonian:

$$
H(\Delta \beta, \psi, \varsigma)=\frac{\beta_{\mathrm{str}}}{\beta_{\mathrm{qs}}^{2}} \cdot \frac{(\Delta \beta)^{2}}{2}+\frac{\beta_{\mathrm{str}}}{\beta_{\mathrm{qs}}} \cdot U(\psi)
$$

with the potential energy:

$$
\begin{aligned}
U(\psi, \varsigma)= & A_{E}\left\{\sin \left(\psi+\bar{\phi}_{s}+\varphi_{a} \sin \nu_{\mathrm{ph}} \varsigma\right)\right. \\
& \left.-\psi \cdot \cos \left(\bar{\phi}_{s}+\varphi_{a} \sin \nu_{\mathrm{ph}} \varsigma\right)\right\} .
\end{aligned}
$$

Taking into account expansion (22) and retaining only a reasonable number of terms, the force $(d \Delta \beta / d \varsigma)$ is represented as

$$
\begin{aligned}
\frac{d \Delta \beta}{d \mathrm{~s}}= & -A_{E} \frac{\beta_{\mathrm{str}}}{\beta_{\mathrm{qs}}} \cdot J_{0}\left(\varphi_{a}\right) \cdot\left[\cos \left(\psi+\bar{\phi}_{s}\right)-\cos \bar{\phi}_{s}\right]+A_{E} \frac{\beta_{\mathrm{str}}}{\beta_{\mathrm{qs}}} \cdot 2 J_{1}\left(\varphi_{a}\right)\left[\sin \left(\psi+\bar{\phi}_{s}\right)-\sin \bar{\phi}_{s}\right] \cdot \cos \nu_{\mathrm{ph}} \varsigma \\
& -A_{E} \frac{\beta_{\mathrm{str}}}{\beta_{\mathrm{qs}}} \cdot 2 J_{2}\left(\varphi_{a}\right)\left[\cos \left(\psi+\bar{\phi}_{s}\right)-\cos \bar{\phi}_{s}\right] \cdot \cos 2 \nu_{\mathrm{ph}} \mathrm{s} .
\end{aligned}
$$

Following the same method [5], the action of a fast oscillating component can be found, when the effective potential is represented through the sum of time-independent and averaged fast oscillating components: 


$$
\begin{aligned}
H(p, \psi)= & \frac{\beta_{\mathrm{str}}}{\beta_{\mathrm{qs}}^{2}} \cdot \frac{(\Delta \beta)^{2}}{2}+A_{E} \frac{\beta_{\mathrm{str}}}{\beta_{\mathrm{qs}}} \cdot J_{0}\left(\varphi_{a}\right) \\
& \cdot\left[\sin \left(\psi+\bar{\phi}_{s}\right)-\psi \cos \bar{\phi}_{s}\right]+A_{E}^{2} \frac{\beta_{\mathrm{str}}^{2}}{\beta_{\mathrm{qs}}^{2}} \cdot \frac{J_{1}^{2}\left(\varphi_{a}\right)}{\nu_{\mathrm{ph}}^{2}} \cdot \sin ^{2}\left(\psi+\bar{\phi}_{s}\right)+A_{E}^{2} \frac{\beta_{\mathrm{str}}^{2}}{\beta_{\mathrm{qs}}^{2}} \frac{J_{2}^{2}\left(\varphi_{a}\right)}{4 \nu_{\mathrm{ph}}^{2}} \cdot \cos ^{2}\left(\psi+\bar{\phi}_{s}\right) .
\end{aligned}
$$

As in the stepped phase velocity structure one can see as a fundamental effect the decreasing of the frequency $\Omega=\sqrt{A_{E}\left(\beta_{\mathrm{str}}^{2} / \beta_{\mathrm{qs}}^{3}\right) J_{0}\left(\varphi_{a}\right)}$ by a factor $\sqrt{J_{0}\left(\varphi_{a}\right)} \approx\left(1-\varphi_{a}^{2} / 8\right)$. The separatrix length remains the same.

The stepped rf phase structure in case $\nu_{\beta} \gg \Omega$ has the same length and a smaller energy spread in the factor $\sqrt{J_{0}\left(\varphi_{a}\right)}$, where the amplitude of oscillation $\varphi_{a}$ is slowly changing in time.

\section{CONCLUSION}

A separatrix formalism for superconducting linear accelerators in the absence of synchronism between particles and phase wave has been developed. The separatrix formalism consists of two parts. The first part is based on the bunch motion relative to a stationary separatrix, and the second part considers acceleration of a bunch in an effective separatrix, moving together with a quasisynchronous particle.

Separatrix formalism has been applied to two options, when the phase velocity changes from cavity to cavity and from family to family. In the first option it is shown how the quasisynchronous phase velocity has to be adjusted by a stepped phase velocity in order to minimize the effect of nonsynchronism. As a result due to the noncompensated part of nonsynchronism the whole bunch makes a coherent oscillation. The amplitude of the coherent oscillation is proportional to the average acceleration rate and inversely proportional to the squared frequency of the phase velocity stepping and is almost constant during acceleration. The acceleration rate is determined by the first time derivative of the averaged structure phase velocity.

In the second option nonsynchronism changes from cavity to cavity and is larger. To create a quasisynchronous motion the stepped rf phasing of cavities is used. It is shown that the amplitude of the coherent oscillation is proportional to the first derivative of the averaged $\mathrm{rf}$ phase and inversely proportional to the frequency of the rf phase stepping. The acceleration rate is determined by the second derivative of the averaged $\mathrm{rf}$ phase change. The amplitude of the coherent oscillation depends on the instantaneous nonsynchronism and changes from cavity to cavity.

In both cases due to the coherent oscillation the momentum spread of the effective separatrix is squeezed proportionally to $\sqrt{J_{0}\left(\varphi_{a}\right)}$, where $\varphi_{m}$ is the coherent oscillation amplitude. Therefore, in the stepped phase velocity structure the effective separatrix momentum spread is almost constant. But in the stepped rf phase structure it can decrease significantly and determines the limit of cavity number in one family. In both options the effective separatrix phase length does not change.

\section{ACKNOWLEDGMENTS}

The authors thank Dr. P. von Rossen for his support.

[1] Yu. Senichev and E. Shaposhnikova, Zh. Tech. Fiz. 57, 36 (1987) [Sov. Phys. Tech. Phys. 32, 632 (1987)].

[2] T. P. Wangler, in Proceedings of the Particle Accelerator Conference, 1999 (IEEE, Piscataway, NJ, 1999), pp. 611613 (http://accelconf.web.cern.ch/AccelConf/p99/papers/ frar2.pdf).

[3] R. Tölle et al., in Proceedings of the European Particle Accelerator Conference, 2002 (EPS-IGA/CERN, Geneva, 2002), pp. 966-968 (http://accelconf.web.cern.ch/AccelConf/e02/papers/thple080.pdf).

[4] L. Landau and E. Lifshitz, Mechanics (Pergamon Press, Oxford, 1969), Vol. I.

[5] N. Bogolyubov and Yu. Mitropol'skij, Asymptotic Methods in the Theory of Non-Linear Oscillations (Hindustan Publishing, Delhi, 1961). 